\title{
Prevalence of Dental Fear and Anxiety and Its Triggering Factors in the Dental Office among School-going Children in
}

\section{Al Ahsa}

\author{
Abdullah F Alshuaibi ${ }^{1}$, Mohammed Aldarwish ${ }^{2}$, Anwar N Almulhim ${ }^{3}$, Gauri S Lele ${ }^{4}$, Suresh Sanikommu ${ }^{5}$, Ravi G Raghunath ${ }^{6}$
}

\begin{abstract}
Aims and objectives:

- To estimate the prevalence of dental fear and anxiety (DFA) among school-going children in Al Ahsa.

- To identify the factors that trigger DFA in the dental office.

Materials and methods: A cross-sectional questionnaire-based study was conducted. Eight hundred and sixteen, 7-12-year-old children studying in government and private schools spread across the city of Al-Ahsa, Saudi Arabia, participated in the study. A self-reported questionnaire with 15 close-ended questions pertaining to DFA in logical order was distributed to all the participants. Each question had three options as responses with images. The children were asked to choose the option that best fitted their response.

Results and Conclusion: The prevalence of DFA among boys and girls was found to be 50.4 and $71.28 \%$, respectively. The majority of the boys studying in government schools reported DFA when informed about the treatment in advance, while most of those studying in private schools reported DFA at the sight of the dental chair and dental injections, while walking into the clinic, in the waiting room, when informed about the treatment in advance and their tooth being drilled. On the contrary, the responses were very similar among girls irrespective of their school. Most of the boys and girls did not experience any DFA when their parents were allowed to accompany them. Hence, a tailor-made approach for the management of the child in the dental office is warranted.

Clinical significance: A tailor-made approach for the management of DFA in the dental office plays a significant role in successful treatment. Keywords: Children, Dental anxiety, Dental fear, Prevalence, School.

International Journal of Clinical Pediatric Dentistry (2021): 10.5005/jp-journals-10005-1925
\end{abstract}

\section{INTRODUCTION}

The terms dental fear and dental anxiety are often used interchangeably in the literature. Hence, dental fear and anxiety (DFA) is the term used to describe all sorts of fears and anxieties related to dentistry. ${ }^{1}$ It is a unique problem with multifactorial etiology affecting all age groups. ${ }^{2,3}$ The environment of the dental clinic, stress-induced while undergoing any procedure, cognitive abilities of the individual, parental attitudes, and cultural practices have been found to have some influence on DFA. ${ }^{4}$

Different studies conducted in different countries have reported that the prevalence of DFA to be $12.5 \%$ in Canada, ${ }^{5} 12.6 \%$ in Russia, ${ }^{6} 13.5 \%$ in France, ${ }^{7} 16.1 \%$ in Australia, ${ }^{8}$ and 30\% in China. ${ }^{6}$ Some studies conducted in Saudi Arabia have reported that DFA among adults is 27 to $51 \%{ }^{4,9}$ Dental fear and anxiety can limit the utilization of oral health services, which in turn affect early detection and management of the disease. ${ }^{7,10,11}$

Dental fear and anxiety among children is not only frustrating for the child and their family but challenging for the dentist as well. Very often, the planned treatment procedures have to be deferred due to behavior management issues. ${ }^{12,13}$ If this gets repeated, it enhances the negative behavior and such patients are extremely difficult to treat by conventional means. Children with higher levels of DFA are likely to have more untreated carious teeth. Thus, it results in poor oral health and an increase in expenses toward dental services. ${ }^{2,7,12}$ A longitudinal study conducted on 7-9-year-old children having DFA has reported that at the age of 7 years, $3 \%$ of children had complained of toothache; $4 \%$ of them had undergone painful treatment. Furthermore, the increase in
${ }_{1,2}$ College of Dentistry, King Faisal University, Al Ahsa, Eastern Province, Kingdom of Saudi Arabia

${ }^{3}$ Department of Dental Clinics Complex, College of Dentistry, King Faisal University, Al Ahsa, Eastern Province, Kingdom of Saudi Arabia

${ }^{4-6}$ Department of Preventive Dental Sciences, College of Dentistry, King Faisal University, Al Ahsa, Eastern Province, Kingdom of Saudi Arabia

Corresponding Author: Ravi G Raghunath, Department of Preventive Dental Sciences, College of Dentistry, King Faisal University, Al Ahsa, Eastern Province, Kingdom of Saudi Arabia, Phone: +91 9948444007, e-mail: gummarajuravi@gmail.com

How to cite this article: Alshuaibi AF, Aldarwish M, Almulhim AN, et al. Prevalence of Dental Fear and Anxiety and Its Triggering Factors in the Dental Office among School-going Children in Al Ahsa. Int J Clin Pediatr Dent 2021;14(2):286-292.

Source of support: Nil

Conflict of interest: None

new carious lesions affecting primary and permanent dentitions was found to be 5 and $7 \%$. On the other hand, among 9-year-old caries affecting primary dentition was found to be $4 \%$ and the increase in new carious lesions affecting primary and permanent dentitions was 5 and $9 \% .^{2}$

The current study was aimed to know the prevalence of dental anxiety and fear in children and to identify the factors that trigger DFA in the dental office. 


\section{Materials and Methods}

\section{Study Design}

A cross-sectional questionnaire-based study was done for 5 weeks from March 5, 2019, to April 12, 2019. Before the commencement of the study, approval from "College Research Review Committee" (CRRC), College of Dentistry, King Faisal University, Al Ahsa (approval No: KFU/CoD/R/0011/2019 DATED 04/04/2019) and permission from the selected schools were obtained.

\section{Study Participants}

Four government and four private schools spread across the city of Al-Ahsa, Saudi Arabia were randomly selected and from each school, all the 7-12-year-old children were selected. Eight hundred and sixteen children from these eight schools participated in the study with their parental consent.

\section{Data Collection}

A 15-item questionnaire was developed in English based on Abeer Children Dental Anxiety Scale (ACDAS). ${ }^{10}$ To ensure content validity, the questionnaire was translated to Arabic by one of the authors with the assistance of an expert who had good command over both languages. Furthermore, the piloting of the questionnaire revealed that there was no need for any changes.

The structured questionnaire in the Arabic language was provided to all the students who had parental consent. Before the distribution of the questionnaire, it was confirmed with the students that they had previous dental visit experience. The purpose and method of filling the questionnaire were clearly explained at the beginning of the session to the participants and all of them completed the form individually. The questionnaire had two sections wherein the initial part had the general information like age, gender, grade, and school and an approval box to provide participants' assent. The second part comprised of a self-reported questionnaire with 15 close-ended questions pertaining to DFA appearing in logical order. Each question had three options with facial images. Facial Image 1 represented the feeling of a relaxed, happy, "not anxious" person. Facial Image 2 represented a fair feeling of being "slightly anxious." Facial Image 3 represented the scary feeling of being "very anxious". The child was asked to choose the best option that best fitted his response. Only completely filled questionnaire forms were considered. Scores 1, 2, and 3 were assigned to Images 1, 2, and 3 respectively. The prevalence of dental anxiety was calculated based on the responses received for all 15 questions. A total score of 15 was considered as "not anxious" while the score 16 and above was "Anxious." All the 816 questionnaires were returned and were found to be completely answered without any missing data. The data were entered into a spreadsheet for further statistical analysis.

\section{Statistical Analysis}

Data were analyzed with Statistical Software for Social Sciences, IBM SPSS version 16. The prevalence of DFA and the difference in the proportion of DFA between boys' and girls' students studying in government and private schools were assessed using the Chisquare test. $p \leq 0.05$ was considered as statistically significant for all analyses.

\section{Results}

Eight hundred and sixteen, 7-12-year-old students studying in government and private schools participated in the study. There was almost an equal representation of boys (49.3\%) and girls (50.7\%). Table 1 represents the prevalence of DFA. It was found that $50.4 \%$ of boys $(N=403)$ and $71.28 \%$ of girls $(N=413)$ were anxious and the differences were statistically significant $(p=0.000)$.

Table 2 represents the factors triggering DFA in the dental operatory. In response to the question, "If you are going to your dentist for treatment tomorrow, how you would feel?" among the boys studying in the government school, 59.5\% reported as not anxious, $32.7 \%$ slightly anxious, and $7.8 \%$ very anxious, whereas in private schools $43.6 \%$ of the children reported as not anxious, $43.6 \%$ slightly anxious, and $12.8 \%$ as very anxious and the differences were statistically significant $(p=0.007)$. However, among girls studying in the government school, $30.4 \%$ were not anxious, $52.2 \%$ were slightly anxious, and $17.3 \%$ were very anxious. Further, in private schools, $39 \%$ of the children were not anxious, $45.5 \%$ were slightly anxious, and $15.4 \%$ were very anxious $(p=0.238)$ and the differences were statistically not significant.

When asked about their feeling while sitting in the waiting room, the majority of the boys reported not anxious (52\% in government and $44 \%$ in private school). $39.2 \%$ of boys studying in government school felt slightly anxious, $7.8 \%$ of them felt very anxious while those studying in private school $40.4 \%$ reported slightly anxious, $15.6 \%$ of them very anxious ( $p=0.046$ ). On the contrary, the majority of the girls felt anxious when made to sit in the waiting room ( $p=0.947)$.

Boys studying in government schools (52.4\%) did not feel any anxiety while walking into the dental clinic area, while $59.2 \%$ of them studying in private school felt anxious $(p=0.024)$. However, the majority of girls did feel anxious while walking into the clinic irrespective of their school of study $(77.2 \%$ in government and $70.7 \%$ in private school; $p=0.351$ ).

When they were made to watch another child being treated $62.1 \%$ of boys from government school were not anxious while $45.6 \%$ from private school were not anxious $(p=0.003)$. In contrast, few of the girls were not anxious (23.9\% in government and $26.8 \%$ in private schools; $p=0.491$ ) while the majority of them felt slightly anxious (56.7\% in government and $50.4 \%$ in private schools). Similar observations were seen when they had to watch their siblings or parents being treated and the differences were statistically not significant ( $p=0.072$ for boys; $p=0.839$ for girls).

Even though, a significant number of boys preferred to be informed in advance about the treatment (48.4\% in government; $40.4 \%$ in private schools) majority of them had anxiety $(p=0.007)$. When informed about the treatment in advance, most of the girls reported slight anxiety $(46.7 \%$ in government and $35 \%$ in private school; $p=0.088$ ).

The sight of a dental chair led to anxiety in girls predominantly ( $p=0.001)$ when compared with boys $(p=0.998)$. Further, the majority of boys studying in private schools $(p=0.003)$ and girls irrespective of the schools ( $p=0.035$ ) were anxious when they saw the dentist in their professional attire. However, $61.4 \%$ of the boys studying government schools did not show any DFA toward dentist's attire.

Table 1: Prevalence of dental anxiety and fear

\begin{tabular}{llllll}
\hline \multicolumn{3}{c}{ Boys $(N=403)$} & & \multicolumn{3}{c}{$\operatorname{Girls}(N=413)$} & \\
\cline { 1 - 2 } \cline { 5 - 6 } $\begin{array}{l}\text { Not anxious } \\
(\leq 15)\end{array}$ & $\begin{array}{l}\text { Anxious } \\
(\geq 16)\end{array}$ & & $\begin{array}{l}\text { Not anxious } \\
(\leq 15)\end{array}$ & $\begin{array}{l}\text { Anxious } \\
(\geq 16)\end{array}$ & p value \\
\hline $20350.4 \%$ & $20049.6 \%$ & & $11928.72 \%$ & $29471.28 \%$ & $0.000^{*}$ \\
\hline
\end{tabular}

*Significant 


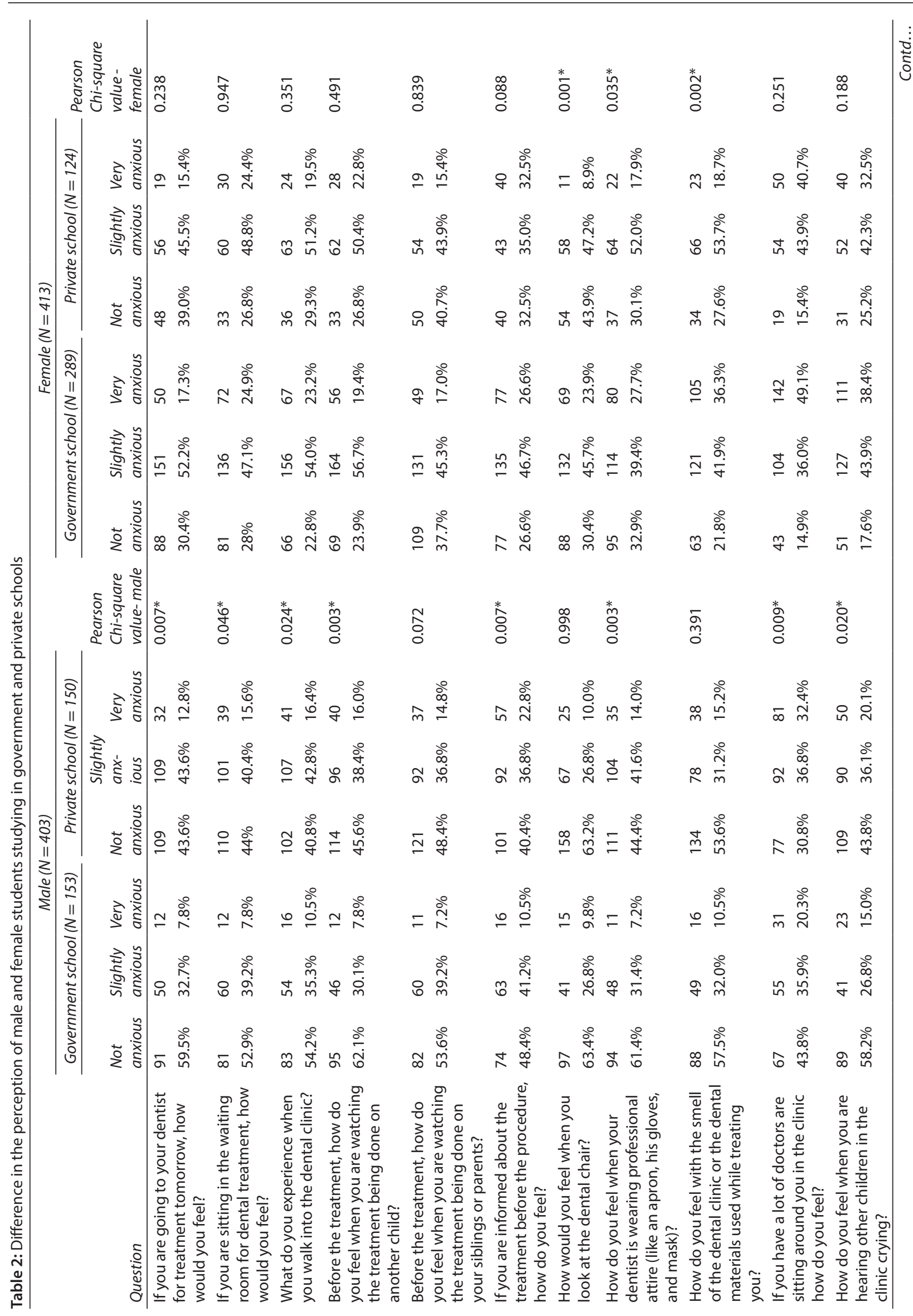




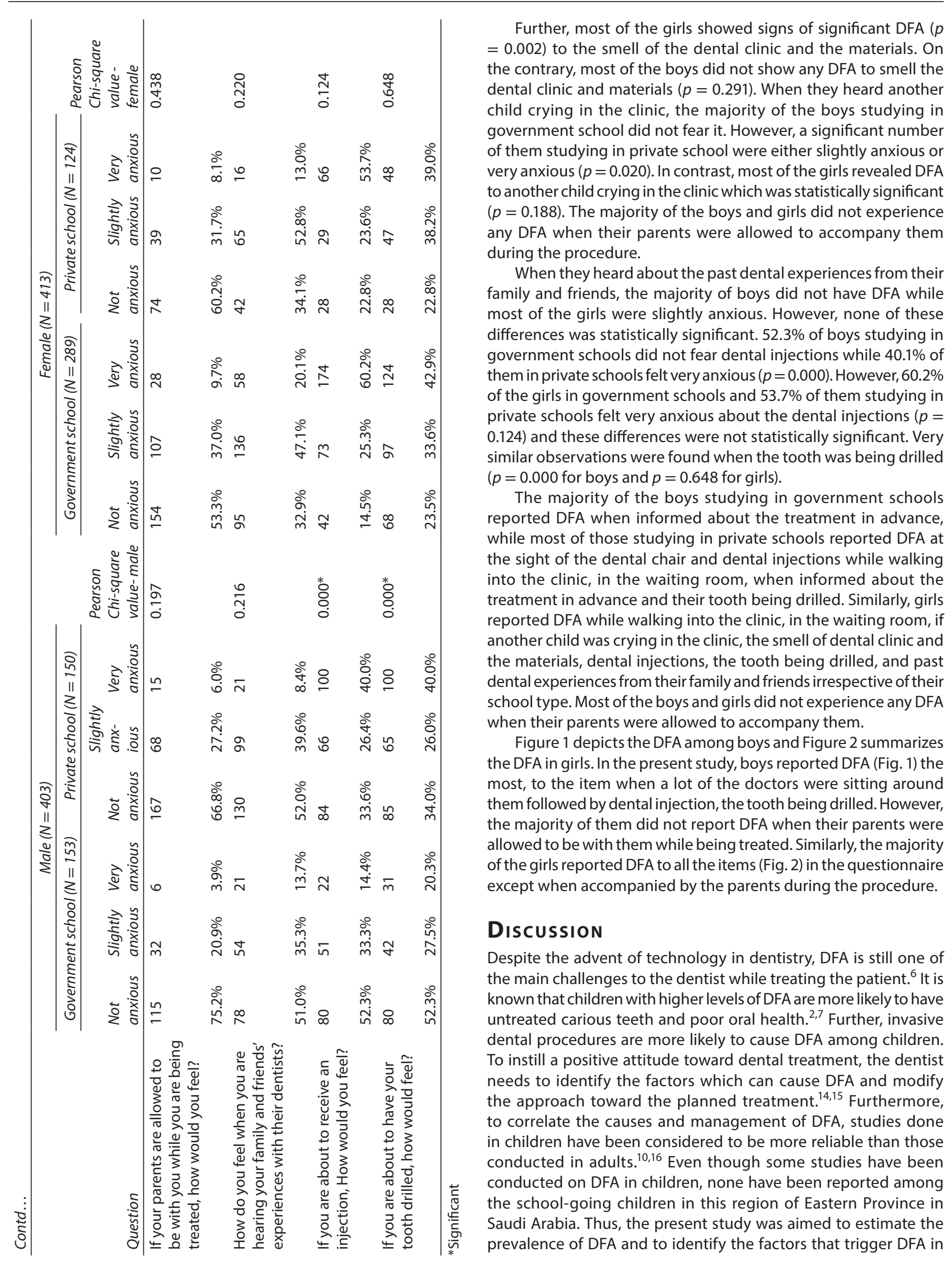




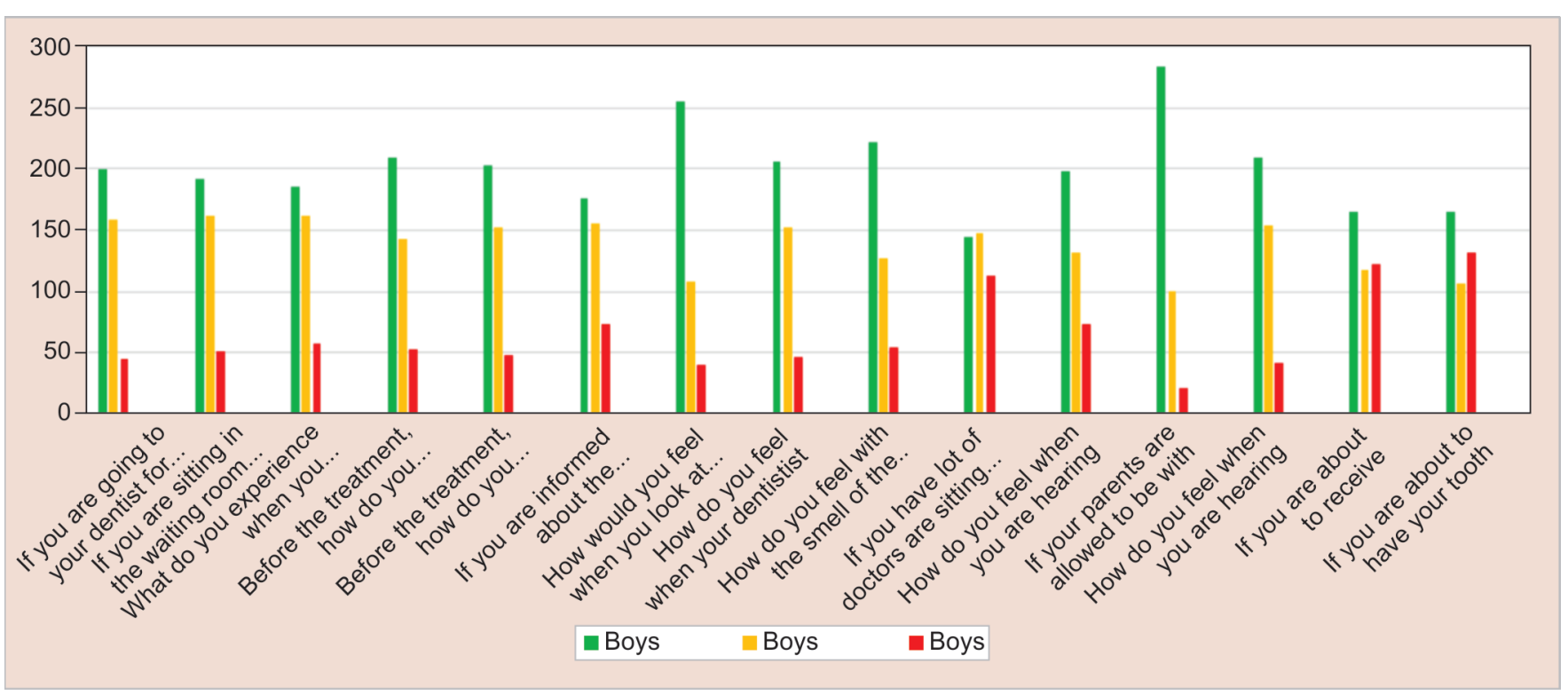

Fig. 1: Dental fear and anxiety among boys

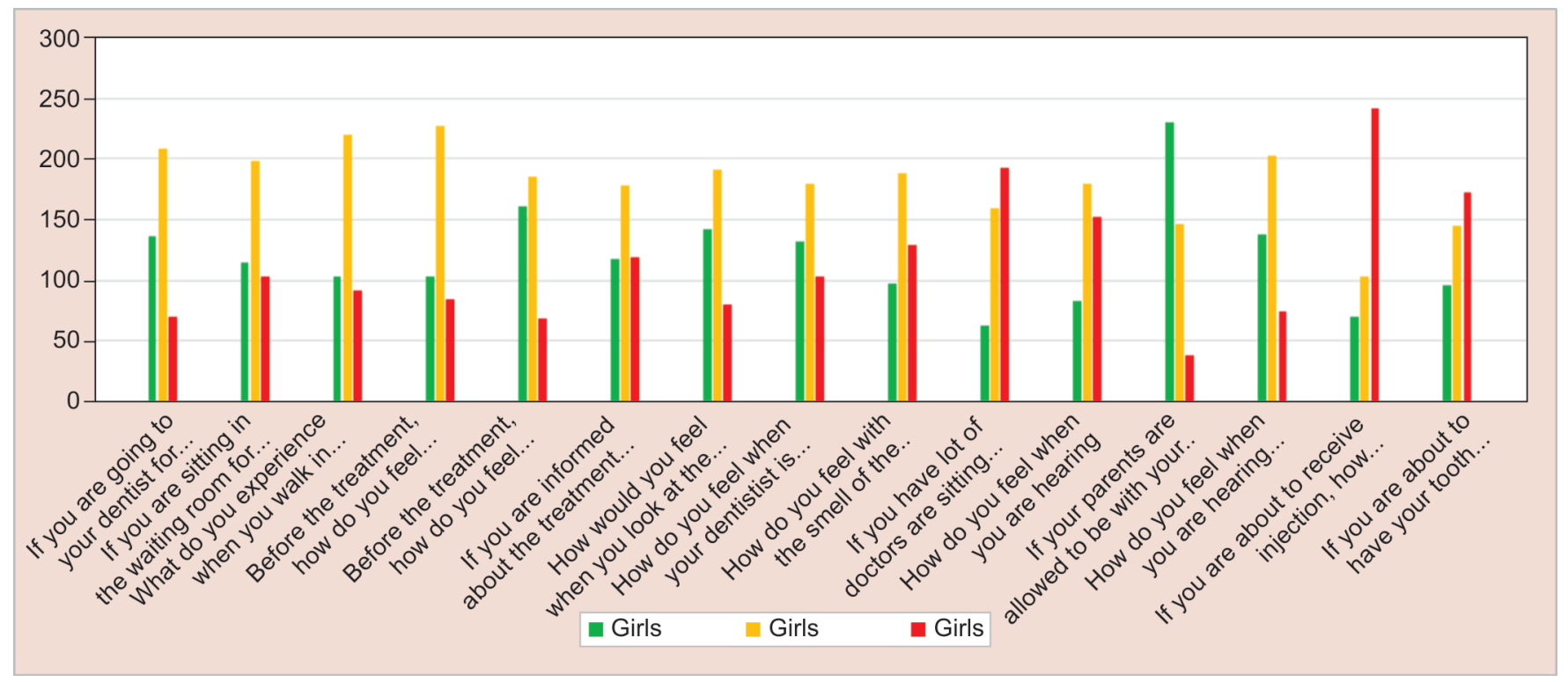

Fig. 2: Dental fear and anxiety among girls

dental operatory among school-going children in Al Ahsa, Eastern Province, Saudi Arabia.

There is no concrete explanation available to describe the development and etiology of DFA in children. Several studies have opined that DFA could be due to multiple factors like age, sociocultural background, parents' and siblings' past dental experiences, or their own experiences. ${ }^{1,6,10,13}$ In the present study, 7-12 years of age children were selected as their cognitive development would enable them to understand the questions and provide appropriate answers. ${ }^{17}$ Many scales such as CDAS, MDAS, CDFSS, Dental Anxiety Inventory (DAI), and FIS are available to assess dental anxiety. However, due to their own limitations, none of these tools have been considered as a gold standard. ${ }^{15,18,19}$

Abeer Children Dental Anxiety Scale being the first dental anxiety scale for children and adolescence has 13 self-reported questions concerned about the feeling of the child's dental experiences. Each question uses facial images which represented the feeling of Happy, OK, or Scared. ${ }^{10,11}$ As the influence of certain new factors was intended to be evaluated the questionnaire was developed. The prevalence of DFA is usually determined by the questions and the assessment tools used. Self-reported scales are often used as they are more reliable even in children who can read and have the cognitive abilities to understand how to report their anxiety on a scale. ${ }^{20-22}$ Considering the anticipated cognitive development among 7- to 12-year-old children, the facial images with the options of "Not anxious", "Slightly anxious", and "Very Anxious" were considered. Thus, 15-item self-reported questionnaire was developed according to the needs of the present study.

The prevalence of DFA was found to be $50.4 \%$ in boys $(N=403)$ and $71.28 \%$ in girls $(N=413)$. Such high prevalence can be attributed to the fact that age is inversely related to dental 
anxiety. ${ }^{9,23}$ It has also been suggested that children who have had previous dental experiences could have higher levels of dental anxiety. ${ }^{13,24,25}$ In addition, a study reported that the difference in DFA among Arabic-speaking and developed countries may be attributed to the differences in culture and organization of healthcare delivery system. In developed countries, preventive measures are emphasized more than curative measures whereas in developing countries most children seek dental treatment only when in pain. ${ }^{1}$ There is no consensus on the relationship between DFA and gender in the available scientific literature as different studies have reported contradicting observations. For example, most studies have concluded that the incidence of DFA is more in girls than boys; while some studies found that boys are more fearful and frequently try to escape during their dental visit. ${ }^{26-29}$ However, few studies have reported that there is no significant relationship between DFA and gender. ${ }^{30,31}$ In the present study, it was found that girls have more DFA than boys.

The differences in study design, sampling, and DFA scales used to make it difficult to draw accurate comparisons with other studies. However, an overview of the factors that trigger DFA among children can be outlined. The majority of the studies have reported that dental injections and extraction as the most fearful procedures followed by "tooth being drilled". ${ }^{1,4,6,8-11}$ In the present study, boys reported DFA (Fig. 1) the most, to the item "when lot of the doctors were sitting around them" followed by dental injection and tooth being drilled. However, the majority of them did not report DFA when their parents were allowed to be with them while being treated. In contrast, the majority of the girls reported DFA to all the items (Fig. 2) in the questionnaire except when accompanied by the parents during the procedure. A lot of doctors sitting around them while being treated were reported to be the most prevalent factor that triggers DFA among girls followed by dental injection and drilling of the tooth. This could be attributed to the fact that in some cultures it is socially acceptable for the girls to express their fear while the boys cannot. ${ }^{32,33}$

Boys also reported DFA when informed about the treatment before the procedure; while walking into the clinic; in the waiting room and when informed about the treatment in advance. On the contrary, girls reported DFA on hearing other children crying in the clinic; the smell of the dental clinic and materials; while walking into the clinic and in the waiting room.

Furthermore in this study, the responses received from the participants studying in the government schools were different as compared to those received from children studying in private schools. For example, the majority of the boys studying in government schools reported DFA when informed about the treatment in advance, while most of those studying in private schools reported DFA at the sight of a dental chair and dental injections while walking into the clinic, in the waiting room, when informed about the treatment in advance and their tooth being drilled. However, the responses were very similar among girls irrespective of their school.

In Saudi Arabia, boys and girls studying in separate schools from primary school and hence social factors affecting DFA may vary among them. ${ }^{1}$ The separation between boys' and girls' peer groups was suggested to be at least a minor factor in African populations. ${ }^{34}$ However, the difference in responses among boys and girls from government and private schools in the present study cannot be considered to imply the role of school per se as a causative or associated factor in triggering DFA. To develop a profound understanding of the variation in DFA among the children from government and private schools, other factors like socioeconomic background, parents' and siblings' past dental experiences, and their own experiences have to be considered.

\section{Conclusion}

This study provides an insight into the factors affecting the DFA that were not investigated previously. The majority of the participants reported DFA. The variations in response to similar factors in the dental office among children enable us to identify those factors that could potentially trigger DFA. Hence, a tailor-made approach for the management of the child in the dental office is warranted to alleviate DFA.

\section{Clinical Significance}

A tailor-made approach for the management of DFA in the dental office plays a significant role in successful treatment.

\section{References}

1. Alshoraim MA, El-Housseiny AA, Farsi NM, et al. Effects of child characteristics and dental history on dental fear: cross-sectional study. BMC Oral Health 2018;18(1):33. DOI: 10.1186/s12903-018-04964

2. Dahlander A, Soares F, Grindefjord M, et al. Factors associated with dental fear and anxiety in children aged 7 to 9 years. Dent J (Basel) 2019;7(3):68.

3. Porritt J, Marshman Z, Rodd HD. Understanding children's dental anxiety and psychological approaches to its reduction. Int J Paediatr Dent 2012;22(6):397-405. DOI: 10.1111/j.1365-263X.2011.01208.x.

4. Fayad MI, Elbieh A, Baig MN, et al. Prevalence of dental anxiety among dental patients in Saudi Arabia. J Int Soc Prev Community Dent 2017;7(2):100-104. DOI: 10.4103/jispcd.JISPCD_19_17.

5. Locker D, Poulton R, Thomson WM. Psychological disorders and dental anxiety in a young adult population. Community Dent Oral Epidemiol 2001;29(6):456-463. DOI: 10.1034/j.1600-0528.2001.290607.x.

6. Kamel AMF, Al-Harbi AS, Al-Otaibi FM, et al. Dental anxiety at Riyadh Elm university clinics. Saudi J Oral Sci 2019;6(2):101-112. DOI: 10.4103/ sjos.SJOralSci_33_19.

7. Nicolas E, Collado V, Faulks D, et al. A national cross-sectional survey of dental anxiety in the French adult population. BMC Oral Health 2007;7(1):12. DOI: 10.1186/1472-6831-7-12.

8. Carter AE, Carter G, Boschen M, et al. Pathways of fear and anxiety in dentistry: A review. World J Clin Cases 2014;2(11):642-653. DOI: 10.12998/wjcc.v2.i11.642Available from: URL: http://www.wjgnet. com/2307-8960/full/v2/i11/642.htm.

9. Gaffar BO, Alagl AS, Al-Ansari AA. The prevalence, causes, and relativity of dental anxiety in adult patients to irregular dental visits. Saudi Med J 2014;35(6):598-603.

10. Al-Namankany A. Assessing dental anxiety in young girls in KSA. J Taibah Univ Med Sci 2018;13(2):123-128. DOI: 10.1016/j. jtumed.2017.11.005.

11. Shafi S, Alasmri A, Mustafa A, et al. An assessment of dental anxiety in nonclinical setting among Saudi Arabian children using Abeer children dental anxiety acale. J Dent Res Rev 2015;2(4):172-174. DOI: 10.4103/2348-2915.176682.

12. Wu L, Gao X. Children's dental fear and anxiety: exploring family related factors. BMC Oral Health 2018;18(1):100. DOI: 10.1186/ s12903-018-0553-zAvailable from: URL: https://bmcoralhealth. biomedcentral.com/articles/.

13. Abanto J, Vidigal EA, Carvalho TS, et al. Factors for determining dental anxiety in preschool children with severe dental caries. Braz Oral Res 2017;31(0):e13. DOI: 10.1590/1807-3107BOR-2017.vol31.0013. 
14. Guinot Jimeno F, Yuste Bielsa S, Cuadros Fernández C, et al. Objective and subjective measures for assessing anxiety in paediatric dental patients. Eur J Paediatr Dent 2011;12(4):239-244.

15. Assuncão CM, Losso EM, Andreatini $R$, et al. The relationship between dental anxiety in children, adolescents and their parents at dental environment. J Indian Soc Pedod Prev Dent 2013;31(3):175-179. DOI: 10.4103/0970-4388.117977.

16. Boman U, Lundgren J, Elfstrom $M$, et al. Common use of fear survey schedule for assessment of dental fear among children and adults. Int J Paediatr Dent 2008;18(1):70-76. DOI: 10.1111/j.1365263X.2007.00863.x.

17. Gaber AE, Khalil AM, Talaat DM, The impact of gender on child dental anxiety in a sample of Egyptian children. (A cross-sectional study) Available from: URL: https://adjalexu.journals.ekb.eg/article_57570 16a0323dffc71384f197df0d0dcd5183.pdf.

18. Appukuttan DP. Strategies to manage patients with dental anxiety and dental phobia: literature review. Clin Cosmet Investig Dent 2016;8:35-50. DOI: 10.2147/CCIDE.S63626.

19. Sousa VEC, Matson J, Dunn Lopez K. Questionnaire adapting: Little changes mean a lot. West J Nurs Res 2017;39(9):1289-1300. DOI: $10.1177 / 0193945916678212$.

20. Al-Namankany A, de Souza M, Ashley P. Evidence-based dentistry: analysis of dental anxiety scales for children. Br Dent J 2012;212(5):219222. DOI: 10.1038/sj.bdj.2012.174.

21. Atkins JrCO, Farrington FH. Informed consent and behavior management. Va Dent J 1994;71(1):16-20.

22. McGrath PA. Measurement issues in research on dental fears and anxiety. Anesth Prog 1986;33(1):43-46.

23. Hmud R, Walsh LJ. Dental anxiety: causes, complications and management approaches. J Mini Intervent 2009;2:67-78.

24. Kruger E, Thomson WW, Poulton R, et al. Dental caries and changes in dental anxiety IN late adolescence. Community Dent Oral Epidemiol 1998;26(5):355-359. DOI: 10.1111/j.1600-0528.1998.tb01973.x.

25. Milsom KM, Tickle M, Humphris GM, et al. The relationship between anxiety and dental treatment experience in 5-year- old children. Br Dent J 2003;194(9):503-506. DOI: 10.1038/sj.bdj. 4810070.

26. Saatchi M, Abtahi M, Mohammadi G, et al. The prevalence of dental anxiety and fear in patients referred to Isfahan Dental School. Iran Dent Res J 2015;12(3):248-253.

27. Nakai $Y$, Hirakawa T, Milgrom $P$, et al. The children's fear survey schedule-dental subscale in Japan. Community Dent Oral Epidemiol 2005;33(3):196-204. DOI: 10.1111/j.1600-0528.2005.00211.x.

28. Arapostathis KN, Coolidge T, Emmanouil D, et al. Reliability and validity of the Greek version of the children's fear survey scheduledental subscale. Int J Paediatr Dent 2008;18(5):374-379. DOI: 10.1111/j.1365-263X.2007.00894.x.

29. Carrillo-Díaz M, Crego A, Armfield J, et al. The moderating role of dental expectancies on the relationship between cognitive vulnerability and dental fear in children and adolescents. Community Dent Oral Epidemiol 2013;41(3):269-278. DOI: 10.1111/cdoe. 12009.

30. Udoye $\mathrm{Cl}$, Oginni AO, Oginni FO. Dental anxiety among patients undergoing various dental treatments in a Nigeria teaching hospital. J Contemp Dent Pract 2005;6(2):91-98. DOI: 10.5005/jcdp-6-2-91.

31. Raj S, Agarwal M, Aradhya K, et al. Evaluation of dental fear in children during dental visit using children's fear survey scheduledental subscale. J Clinic Pediatr Dent 2013;6(1):12-15. DOI: 10.5005/ jp-journals-10005-1178.

32. El-Housseiny AA, Alamoudi NM, Farsi NM, et al. Characteristics of dental fear among Arabic-speaking children: a descriptive study. BMC Oral Health 2014;14(1):118. DOI: 10.1186/1472-6831-14-118.

33. Economou GC. Dental anxiety and personality: investigating the relationship between dental anxiety and self-consciousness. J Dent Educ 2003;67(9):970-980. DOI: 10.1002/j.0022-0337.2003.67.9.tb036 95.x.

34. Folayan MO, Idehen EE, Ojo OO. The modulating effect of culture on the expression of dental anxiety in children: a literature review. Int J Paediatr Dent 2004;14(4):241-245. DOI: 10.1111/j.1365263X.2004.00563.x. 